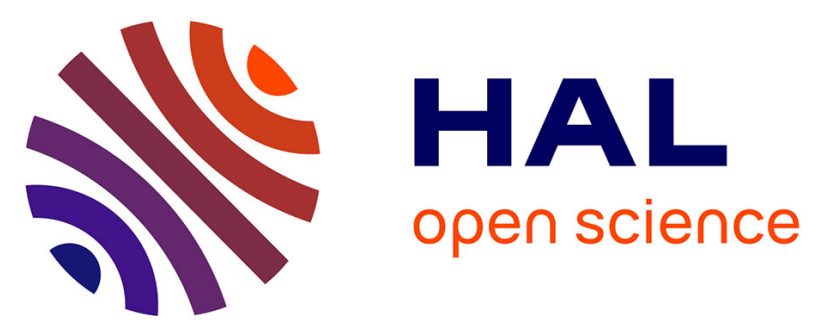

\title{
Predicting Progression to Advanced Age-Related Macular Degeneration from Clinical, Genetic, and Lifestyle Factors Using Machine Learning
}

Soufiane Ajana, Audrey Cougnard-Grégoire, Johanna Colijn, Bénédicte M.J. Merle, Timo Verzijden, Paulus T.V. M. de Jong, Albert Hofman, Johannes

Vingerling, Boris Hejblum, Jean-François Korobelnik, et al.

\section{To cite this version:}

Soufiane Ajana, Audrey Cougnard-Grégoire, Johanna Colijn, Bénédicte M.J. Merle, Timo Verzijden, et al.. Predicting Progression to Advanced Age-Related Macular Degeneration from Clinical, Genetic, and Lifestyle Factors Using Machine Learning. Ophthalmology: Journal of The American Academy of Ophthalmology, 2021, 128 (4), pp.587-597. 10.1016/j.ophtha.2020.08.031 . hal-03100320

\section{HAL Id: hal-03100320 \\ https://hal.inria.fr/hal-03100320}

Submitted on 7 Jan 2021

HAL is a multi-disciplinary open access archive for the deposit and dissemination of scientific research documents, whether they are published or not. The documents may come from teaching and research institutions in France or abroad, or from public or private research centers.
L'archive ouverte pluridisciplinaire HAL, est destinée au dépôt et à la diffusion de documents scientifiques de niveau recherche, publiés ou non, émanant des établissements d'enseignement et de recherche français ou étrangers, des laboratoires publics ou privés. 
Predicting progression to advanced age-related macular degeneration from clinical, genetic and lifestyle factors using machine learning

Authors: Soufiane Ajana PhD, ${ }^{1}$ Audrey Cougnard-Grégoire PhD,${ }^{1 *}$ Johanna M Colijn M.D., MSc,,$^{2,3^{*}}$ Bénédicte MJ Merle PhD,,$^{*}$ Timo Verzijden MSc, ${ }^{2,3}$ Paulus TVM de Jong M.D., PhD, ${ }^{4,5}$ Albert Hofman $\mathrm{PhD},{ }^{3,6}$ Johannes R Vingerling M.D., PhD, ${ }^{2}$ Boris $\mathrm{P}$ Hejblum PhD, ${ }^{1,7}$ Jean-François Korobelnik M.D., ${ }^{1,8}$ Magda A Meester-Smoor PhD, ${ }^{2,3}$ Marius Ueffing ${ }^{9}$, Hélène Jacqmin-Gadda PhD,$^{1^{* *}}$ Caroline CW Klaver M.D., PhD, ${ }^{2,3,10,}$ $1^{1 * *}$ and Cécile Delcourt PhD ${ }^{1 * *}$ for the EYE-RISK Consortium ${ }^{\S}$

${ }^{1}$ Univ. Bordeaux, Inserm, Bordeaux Population Health Research Center, UMR 1219, Bordeaux, France.

2 Department of Ophthalmology, Erasmus University Medical Center, Rotterdam, The Netherlands.

${ }^{3}$ Department of Epidemiology, Erasmus University Medical Center, Rotterdam, The Netherlands.

${ }^{4}$ Department of Retinal signal processing, Netherlands Institute of Neurosciences, KNAW; Department of Ophthalmology, Amsterdam University MC, Amsterdam, The Netherlands.

${ }^{5}$ Department of Ophthalmology, Leiden University MC, Leiden, The Netherlands.

${ }^{6}$ Department of Epidemiology, Harvard T.H. Chan School of Public Health, Boston, MA, USA.

${ }^{7}$ Inria, SISTM, Bordeaux Sud-Ouest, Bordeaux, France.

${ }^{8}$ Department of Ophthalmology, Bordeaux University Hospital, Bordeaux, France.

${ }^{9}$ Department of Ophthalmology, Institute for Ophthalmic Research, University of Tübingen,Tübingen, Germany

${ }^{10}$ Department of Ophthalmology, Radboud University Medical Center, Nijmegen, The Netherlands.

${ }^{11}$ Institute of Molecular and Clinical Ophthalmology Basel, Basel, Switzerland. equally to this work and are considered co-second authors. equally to this work and are considered co-last authors. 
$\S$ Members of the EYE-RISK consortium are listed in annex.

Corresponding author: Cécile Delcourt, "lifelong exposure health and aging" team, Research Center Inserm U1219, Université de Bordeaux - ISPED, 146, rue Léo Saignat, CS61292, 33076 Bordeaux Cedex. Email: cecile.delcourt@u-bordeaux.fr

This article contains additional online-only material. The following should appear online-only: Figures S1, S2 and S3 and Table S1.

Financial support: The EYE-RISK project was supported by the European Union's Horizon 2020 Research and Innovation Programme (grant no.: 634479). The Rotterdam Study is funded by Erasmus Medical Center and Erasmus University, Rotterdam, The Netherlands; the Organization for the Health Research and Development (ZonMw); the Research Institute for Diseases in the Elderly (RIDE); the Ministry of Education, Culture and Science; the Ministry for Health, Welfare and Sports; the European Commission (DG XII), and the Municipality of Rotterdam,

Rotterdam, The Netherlands. The funders had no role in study design, data collection and analysis, decision to publish, or preparation of the manuscript.

Additionally, the ophthalmic research within the Rotterdam Study was supported by the following foundations: Oogfonds; Bartiméus Sonneheerdt Vereniging; Landelijke Stichting voor Blinden en Slechtzienden; Algemene Nederlandse Vereniging Ter Voorkoming Van Blindheid; Novartis Foundation; and MaculaFonds, which contributed through UitZicht (grant nos.: 2015-36 and 2016-19). The Antioxydants, 
Lipides Essentiels, Nutrition et Maladies Oculaires (ALIENOR) Study was funded by Laboratoires Théa; Fondation Voir et Entendre; Retina France; Agence Nationale de la Recherche (ANR 2010-PRSP-011 VISA); Programme Hospitalier de Recherche Clinique (PHRC) (ECLAIR project-2012) ; CFSR Recherche, the French Speaking Retina Specialists' Club and Caisse Nationale pour la Solidarité et l'Autonomie.

The funders had no role in study design, data collection and analysis, decision to publish, or preparation of the manuscript. Laboratoires Théa participated in the design of the Antioxydants, Lipides Essentiels, Nutrition et Maladies Oculaires (ALIENOR) Study, but none of the sponsors of this study participated in the collection, management, statistical analysis, or interpretation of the data, or in the preparation, review, or approval of the present manuscript.

Running head: Predicting progression to advanced age-related macular degeneration 


\section{Abstract:}

Objective: Current prediction models for advanced age-related macular degeneration (AMD) are based on a restrictive set of risk factors. The objective of this study was to develop a comprehensive prediction model, applying a machine learning algorithm allowing selection of the most predictive risk factors automatically.

Design: Two population-based cohort studies

Participants: The Rotterdam Study I (RS-I, training set) included 3838 participants aged 55 years or more, with a median follow-up period of 10.8 years and 108 incident cases of advanced AMD. The ALIENOR study (test set) included 362 participants aged 73 years or more, with a median follow-up period of 6.5 years and 33 incident cases of advanced AMD.

Methods: The prediction model used the bootstrap lasso for survival analysis to select the best predictors of incident advanced AMD in the training set. Predictive performance of the model was assessed using the area under the receiver operating characteristic curve (AUC).

Main outcome measures: incident advanced AMD (atrophic and/or neovascular), based on standardized interpretation of retinal photographs.

Results: The prediction model retained i) age, ii) a combination of phenotypic predictors (based on the presence of intermediate drusen, hyper-pigmentation in one or both eyes and age-related eye disease study (AREDS) simplified score), iii) a summary genetic risk score based on 49 single nucleotide polymorphisms, iv) smoking, v) diet quality, vi) education, and vii) pulse pressure. The cross-validated AUC estimation in RS-I was 0.92 [0.88-0.97] at 5 years, 0.92 [0.90-0.95] at 10 years and $0.91[0.88-0.94]$ at 15 years. In ALIENOR, the AUC reached 0.92 at 5 years 
62 [0.87-0.98]. In terms of calibration, the model tended to underestimate the cumulative 63 incidence of advanced AMD for the high-risk groups, especially in ALIENOR.

Conclusions: This prediction model reached high discrimination abilities, paving the way towards making precision medicine for AMD patients a reality in the near future. 
Age related macular degeneration (AMD) is a leading cause of blindness, worldwide. ${ }^{1}$ It is a multifactorial degenerative eye disease affecting the central part of the retina, named macula. ${ }^{2}$ Its prevalence is rising alongside the general ageing of the human population $^{3}$, with a projected number of individuals affected by AMD worldwide of 196 million in 2020 and up to 288 million in $2040 .{ }^{4}$ AMD typically progresses through different stages from an early to an advanced form, subdivided into atrophic and neovascular subtypes. Advanced AMD results in permanent severe visual impairment or blindness, with major consequences on quality of life and functional independence. ${ }^{2}$ Major therapeutic progress has been made for the neovascular form of the disease in the last 15 years, with the intravitreal injection of anti-angiogenic agents. ${ }^{2}$ However, these treatments are not curative, represent a major cost for society (for instance 2.3 billion of dollars annually for Medicare beneficiaries) and do not always successfully stave off vision loss. ${ }^{5}$ Moreover, there still is no treatment available for the atrophic form of the disease (about $50 \%$ of advanced cases).

Prediction models are increasingly being used by clinicians in order to stratify patients according to their risk and adapt therapies, recommendations and follow-up frequency. Such models can also be used for patient selection in clinical trials testing new therapies or interventions. For instance, several prediction models are now widely used in clinical practice for cardiovascular disease risk assessment. ${ }^{6}$ Both genetic and modifiable risk factors (in particular smoking, nutrition and vascular risk factors) for advanced AMD have been previously identified ${ }^{7}$, and several prediction models have been proposed. ${ }^{8-14}$ However, these prediction models did not include dietary factors, relied only on a limited set of lifestyle factors and incorporated only a restricted set of genetic variants. 
Furthermore, stepwise selection methods are still widely used to develop such models despite their lack of stability ${ }^{15,16}$ and the availability of more principled alternatives. In particular, penalized regression methods can handle overfitting and multicollinearities among the predictors and have been proven to improve stability of variable selection as well as prediction performance. ${ }^{17,18}$ In addition, in elderly cohorts, death is a competing event for the onset of advanced AMD. But due to interval censoring (AMD is assessed only at follow-up visits in the cohort), AMD status at death is unknown for subjects without advanced AMD at their last visit. Standard survival analyses (e.g. Cox model) thus underestimate the incidence of advanced AMD because they ignore advanced AMD cases developing between the last visit and death. More importantly, regression parameters from Cox model may be biased if the excess risk of death among AMD patient is large and predictors of AMD are also associated with death risk. ${ }^{19}$

The aim of this article is to develop a prediction model for advanced AMD from a wider set of potential predictors. Thus, our prediction model was developed from a population-based cohort using a machine learning (ML) approach performing both parameter estimation and automatic variable selection simultaneously. In addition, this prediction model was validated on an independent population-based cohort to better assess its generalizability.

\section{Methods}

\section{Study design and participants}

The training data set included participants enrolled in the Rotterdam Study I (RS-I). The validation dataset included participants enrolled in the Antioxydants, LIpids 
Essentiels, Nutrition et maladies OculaiRes (ALIENOR) study. The complete methodologies of these two population-based cohorts have been detailed elsewhere. 20, 21 Data from both studies were harmonized and included in the database of the EYE-RISK consortium (www.eyerisk.eu) which aims at proposing new clinical guidelines and recommendations for prevention, and at providing a better understanding of the pathogenetic causes of AMD. ${ }^{3}$ All participants gave written informed consent for participation in accordance with the declaration of Helsinki and ethics approval was obtained in both studies.

RS-I included participants aged 55 and over. Baseline examinations took place from 1990 to 1993 and 4 follow-up examinations were performed in 1993-1995, 1997 1999, 2002-2004 and 2009-2011.

ALIENOR included participants aged 73 years or more at first eye examination. Baseline eye examinations took place from 2006 to 2008 and 4 follow-up examinations were performed in 2009-2010, 2011-2012, 2013-2015 and 2015-2017.

\section{Main outcome}

Classification of advanced AMD (neovascular or atrophic AMD) was based on retinal photographs, interpreted by trained graders according to a modification of the Wisconsin Age-Related Maculopathy Grading System ${ }^{22}$ for RS-I and according to the International Classification ${ }^{23}$ for ALIENOR. These classifications were identical regarding for advanced AMD, but showed some differences regarding features of early AMD (such as drusen area or location, for instance). Only features using identical definitions in both classification were used in the analyses. 
Incidence of advanced AMD was defined as participants without advanced AMD at baseline progressing to advanced AMD at any point during the study period. All advanced AMD cases were adjudicated by retina specialists of the corresponding study and harmonized within the EYE-RISK consortium. ${ }^{3}$

\section{Statistical analysis}

We compared excluded participants with those included in the final set, both in RS-I and ALIENOR using the t-test for continuous variables and the chi-square test or the Fisher exact test for categorical variables accordingly. All the p-values were adjusted for multiple testing using the Holm correction method (controlling the Family Wise Error Rate). ${ }^{24}$

The training dataset comprised a set of 33 variables which were common to RS-I and ALIENOR: 7 variables describing retinal alterations (including the AREDS simplified scale,$^{25}$ the Beckman classification ${ }^{26}$ and the Rotterdam classification $\left.{ }^{27}\right), 25$ variables for demographic, lifestyle (including the Mediterranean diet score ${ }^{28}$ ) and comorbidities, and a genetic risk score based on 49 previously identified single nucleotide polymorphisms (SNPs). Based on the 52 single nucleotide polymorphisms (SNPs) proposed by Fritsche et al. ${ }^{29}$, the genetic risk score was calculated only for participants without missing data for the following 5 major risk alleles:

CFH_rs10922109, CFH_rs570618, C2_rs11603772 (merged with: C2_rs429608), C3_rs2230199 and ARMS2_rs3750846. In addition, 3 minor alleles with high missingness were excluded from the analyses: TRPM3_rs71507014, CNN2_rs67538026 and MMP9_rs142450006. Thus, the final genetic risk score was 
calculated as a linear combination of 49 SNPs using the beta coefficients of Fritsche et al. study. ${ }^{29}$

We performed variable selection using the lasso method in a survival framework. ${ }^{30}$ However, since the lasso may not be consistent in terms of variable selection when there is a strong correlation between the relevant and the irrelevant predictors ${ }^{30,31,31,}$ ${ }^{32}$ we insured the stability of the selected predictors using the bootstrap as recommended by several authors. ${ }^{33,34}$ As shown in Figure 1, we started by performing a resampling with replacement from the entire Rotterdam Study I (RS-I) cohort in order to generate 100 bootstrap samples. Of note, we stratified the individuals in each bootstrap sample according to the incidence rate of advanced AMD. The objective was to maintain the same ratio of events in each generated bootstrap sample since there is a low number of events in RS-I (2.8\%). We then estimated a lasso in a survival framework ${ }^{30}$ in each bootstrap sample in order to perform variable selection while handling the multicollinearity of some predictors. The penalization parameter of the lasso was estimated by minimizing the partial likelihood deviance using a 5-fold cross-validation stratified on the incidence rate of advanced AMD. The final set of selected variables was composed of the most frequently selected variables on all the bootstrap samples using the elbow criterion. ${ }^{35}$

The risk score was then estimated by including the final set of selected variables in a semi-parametric Illness-Death Model (IDM) (i.e., the baseline transition intensities were left unspecified and approximated by M-splines) to account for the probability of developing advanced AMD between the last visit without the disease and death. ${ }^{36}$ The IDM was estimated with the R package smoothHazard v1.4.1. ${ }^{37}$ The parameters of the IDM were estimated by maximization of the penalized likelihood. The penalization parameters, which control the trade-off between the data fit and the 
smoothness of the intensity functions, were estimated by leave-one-out crossvalidation. We selected an equidistant sequence of 5 knots between the minimal and maximal event times to fit the splines for each transition intensity. Finally, we included the variables selected by bootstrap lasso as inputs for the regression model of the transition intensity to advanced AMD and we added gender (in addition to the variables selected by bootstrap lasso) as input for the regression models of the two transition intensities to death.

The risk score for advanced AMD was defined as the linear predictor for the transition intensity to advanced AMD estimated from this IDM. We then defined 2 cutpoints at 5 years in RS-I, in order to classify participants in 3 risk categories (low, intermediate and high). The first threshold was chosen to be highly sensitive (sensitivity closest to 95\%) and the second to be highly specific (specificity closest to $95 \%)$ similarly to Delcourt et al. ${ }^{38}$

Finally, prediction performance was evaluated both internally in RS-I (using crossvalidation) as well as externally in ALIENOR. Discrimination performance was assessed by calculating the area under the receiver operating curve (AUC) for incident advanced AMD while accounting for the competing risk of death. ${ }^{39}$ All AUCs are reported with $95 \%$ confidence intervals, based on the quantiles of the standard normal distribution. ${ }^{39}$ Calibration was evaluated by comparing the observed cumulative incidence to the model-estimated cumulative incidence of advanced AMD in each risk category. Of note, the predicted CIF of advanced AMD was estimated using a parametric IDM where the baseline transition intensities were modelled by Weibull distributions. The observed CIF was estimated using a semi-parametric IDM where the baseline transition intensities were left unspecified and approximated by M-splines. ${ }^{36}$ 
In a sensitivity analysis, other missing data for comorbidities, phenotypic and environmental variables were imputed using multiple imputation by chained equations. ${ }^{40}$ In RS-I, participants for whom either all genetic data $(n=572)$ or all nutritional data $(n=768)$ was missing were excluded from the training set, because it became impossible to build a reliable imputation model in such cases. We generated 5 imputed datasets and the imputation procedure was iterated 20 times. We performed all the analyses using $\mathrm{R}$, version 3.3.4 (R Foundation). We used two-sided $\mathrm{P}$ values with an $\alpha=.05$ threshold for statistical significance.

\section{Results}

\section{Study participants}

As shown in Figure 2, among the 5,214 RS-I participants without advanced AMD at baseline and with at least one follow-up examination, 572 had no genetic data, mostly because of lack of blood samples, 144 participants had incomplete phenotypic data and 690 had incomplete lifestyle data (including comorbidities, nutritional and environmental data), leading to a final sample size of 3,838 subjects and 108 cases of incident advanced AMD. Among the 617 participants of the ALIENOR study with incidence data and without advanced AMD at baseline, 112 had no genetic data, 107 had incomplete phenotypic data and 36 had incomplete lifestyle data, leading to a final sample size of 362 participants and 33 cases of incident advanced AMD.

The description of the included and excluded participants of both cohorts is provided in Table S1 (available at http://www.aaojournal.org). In both RS-I and ALIENOR study, included participants tended to be younger than those excluded. Additionally, in RS-I, the included participants tended to have less frequently early AMD, to have 
more frequently secondary education and to take less frequently anti-diabetic drugs. Furthermore, included participants of RS-I were 10 years younger on average than those of ALIENOR — they were less frequently affected by early AMD and less frequently used anti-diabetic and lipid lowering drugs. They were also more often smokers, and adhered less to the Mediterranean diet. Interestingly, the incidence of advanced AMD was higher in ALIENOR compared to RS-I, especially in the first 2 years of follow-up (see Figure S1, available at http://www.aaojournal.org).

\section{Prediction model}

The 33 variables in the analysis and the results of the variable selection obtained by the bootstrap lasso technique are presented in Figure 3. Using the elbow criterion, we selected the first 9 variables as being the most predictive of incident advanced AMD. Particularly, 4 variables (presence of intermediate drusen, genetic risk score, AREDS simplified scale and age) were selected in $100 \%$ of the 100 bootstrap samples. In addition, smoking, pulse pressure (the difference between systolic and diastolic blood pressure), presence of hyperpigmentation, education and the Mediterranean diet score were selected in more than $80 \%$ of the bootstrap samples, while other variables were selected less frequently. Interestingly, these 9 variables were also the most frequently selected in the 5 imputed datasets.

Our prediction model reached very high discrimination performances (Figure S2, available at http://www.aaojournal.org). In RS-I, the cross-validated AUCs were 0.92 (95\% Cl, 0.88 to 0.97$), 0.92(95 \% \mathrm{Cl}, 0.90$ to 0.95$)$ and $0.91(95 \% \mathrm{Cl}, 0.88$ to 0.94$)$ at 5, 10 and 15 years of follow-up, respectively. In ALIENOR, the AUC was 0.92 (95\% $\mathrm{Cl}, 0.87$ to 0.98$)$ at 5 years of follow-up. 
As genetic testing is not currently available in routine ophthalmological practice, we tested the prediction performance of an IDM model with 8 variables (after exclusion of the genetic risk score). In the Rotterdam study, the cross-validated AUCSs were $0.91(95 \% \mathrm{Cl}, 0.86-0.95), 0.90(95 \% \mathrm{Cl}, 0.87-0.93)$ and $0.88(95 \% \mathrm{Cl}, 0.84-0.92)$ at 5 , 10 and 15 years of follow-up, respectively. In ALIENOR, the AUC was $0.93(95 \% \mathrm{Cl}$, 0.89-0.97).

\section{Risk categories characteristics}

We described the distribution of the risk score calculated in RS-I and validated in ALIENOR for incident advanced AMD and non-incident advanced AMD participants (Figure S3, available at http://www.aaojournal.org). As expected, we observe a bimodal distribution in both studies, although some overlap persisted.

To further investigate the prediction ability of the final prediction model, we classified the participants in 3 risk categories (low, intermediate and high). The definition of the cut-points was performed in RS-I. The first cut-point was set at 1.49 , corresponding to a sensitivity of $93.8 \%$, and the second cut-point was set at 2.73 , corresponding to a specificity of $95.0 \%$.

We estimated the cumulative incidence of advanced AMD in each of the risk categories for RS-I and ALIENOR using a semi-parametric IDM (Figure 4). In both samples, the high-risk group was characterized by a high cumulative incidence increasing steeply from baseline and reaching $21 \%$ at 8 years and $44 \%$ at 15 years in RS-I and $32 \%$ at 8 years in ALIENOR. In both samples, the intermediate-risk and the low-risk categories showed much lower incidence rates than the higher risk category, at all-time points. Particularly, the low-risk category showed a cumulative incidence close to 0 during the whole follow-up in both cohorts. In RS-I, the incidence in the intermediate-risk and low-risk groups were distinguishable only in the long term 
(after 5 years). In ALIENOR, both incidences remained very close until 8 years of follow-up. This is probably due to the very low number of events in both of these categories (see Table 1). It is however to be noted that in RS-I, the incidence of advanced AMD in the intermediate group was only after 7-10 years clearly higher than that in the low risk category.

As shown in Table 1, in RS-I, 3,146 (81.9\%) participants were classified as low-risk, $405(10.6 \%)$ as intermediate-risk and $287(7.5 \%)$ as high-risk. Overall, $49 \%$ of the incident cases were observed in the high-risk category versus $24 \%$ in the intermediate-risk category and $27 \%$ in the low-risk category (due to the larger size of this last group). The AMD genetic risk score ranging from -3.36 to 4.54 in the whole sample had a mean of $0.2,1.2$ and 1.6 in the low-risk, intermediate-risk and high-risk categories respectively. Regarding phenotypic characteristics, $93.3 \%$ of participants in the low-risk category were classified as free of AMD in the AREDS simplified scale (score 0 ). Interestingly, this was also the case for $73.1 \%$ and $26.1 \%$ of the participants classified in the intermediate-risk and high-risk groups, respectively. The high-risk participants with an AREDS score of 0 were characterized by a high frequency of intermediate drusen at baseline (84\%), a very high genetic risk score (mean of 2.06) and a high cumulative incidence of advanced AMD (9 incident cases out of $75,12 \%)$. Finally, regarding lifestyle and co-morbidities, the high-risk category showed a higher frequency of primary education, a lower adherence to the Mediterranean diet score and a higher mean pulse pressure compared to the intermediate-risk and low-risk groups. 
In ALIENOR, $52.5 \%$ of the participants were classified as low-risk, $22.3 \%$ as intermediate-risk and $25.2 \%$ as high-risk. Overall, $81.8 \%$ of the incident cases were observed in the high-risk category, $6.1 \%$ in the intermediate-risk category and $12.1 \%$ in the low-risk category. Again, $15.4 \%$ of the participants classified as high-risk had a score of 0 on the AREDS simplified scale. Regarding lifestyle and co-morbidities, we observed the same trends as in RS-I.

Finally, we assessed the calibration of the prediction model (i.e., the ability of the model to correctly predict the incidence of advanced AMD in each risk category). As displayed in Figure 5, the model tends to underestimate the cumulative incidence of advanced AMD for the high-risk groups, especially in ALIENOR.

\section{Discussion}

We developed a prediction model for advanced AMD based on phenotypic, genotypic and lifestyle factors. This model reached high discrimination performances with an AUC equal to $92 \%$ at 5 years in the external validation set. In addition, the proposed model showed a good stratification of participants into low-risk and high-risk groups of incident advanced AMD both in RS-I and ALIENOR.

Buitendijk et al. developed a prediction model for incident advanced AMD based on RS-I and validated it in two independent population-based cohorts. ${ }^{10}$ Other prediction models for incident advanced AMD trained on the Age-Related Eye Disease Study (AREDS) clinical trial were proposed 8, 9, 12, 14, 41 These models were similarly based on genetic, phenotypic and lifestyle variables and also reached high discrimination performances externally (AUC of 0.85 to 0.90 ), although somewhat lower than the model presented here. The main differences with the present model lie in the 
inclusion of a wider set of lifestyle and comorbidities factors as well as a genetic score based on 49 AMD-related SNPs. To our knowledge, the present study is the first to use an automatic variable selection approach to select the most predictive variables from a wide set of predictors. ${ }^{33}$ Indeed, this is a principled way to identify new predictors compared to less rigorous strategies used in the aforementioned prediction models where only a limited set of variables were selected a priori. Of particular interest, among the predictors selected by the bootstrap lasso approach here, pulse pressure as well as the Mediterranean diet score were never included in the previously reported AMD prediction models. These predictors have previously been reported as associated with AMD and are modifiable factors, which could lead to preventive interventions. ${ }^{42-44}$ Interestingly, the bootstrap lasso also selected education, which we believe is more likely to be a surrogate for other unmeasured lifestyle risk factors rather than a direct risk factor of advanced AMD. We also considered the competing risk of death during both the training of the model ${ }^{36}$ and when assessing its discrimination performance ${ }^{39}$, which was not done in previous studies.

Furthermore, in order to insure comparability with the previously developed prediction models, we ran a standard Cox model (instead of an IDM) on the variables selected by bootstrap lasso, considering subjects who died without AMD as censored at their last visit before death. This "naïve" model reached slightly better predictive discrimination performances than the IDM with a cross-validated AUC in RS-I equal to $0.94(95 \% \mathrm{Cl}, 0.89$ to 0.99$)$ at 5 years, $0.93(95 \% \mathrm{Cl}, 0.90$ to 0.96$)$ at 10 years and $0.92(95 \% \mathrm{Cl}, 0.89$ to 0.95$)$ at 15 years. In ALIENOR, the AUC remained at 0.92 at 5 years $(95 \% \mathrm{Cl}, 0.87$ to 0.97$)$. The differences in AUC between the Cox and IDM models were small, most probably because the risk of death was not much higher 
among AMD patients and only a few of the selected predictors for AMD were predictors of death. ${ }^{19}$

Since genetic testing is not currently available in routine ophthalmological practice, we also ran an IDM model without the genetic risk score. Although the genetic risk score was selected in $100 \%$ of the boostrap lasso samples, the AUCs of this model were similar to those obtained with the complete model both in the Rotterdam $(91 \%$, 90\% and $88 \%$ at 5,10 and 15 years, respectively) and Alienor studies (93\% at 5 years). However, in a minority of cases, genetic information might help identify high risk subjects. In particular, a small number of subjects with a simplified AREDS score $\underline{0 \text { were classified as high risk. They had a high genetic score (average 2.06) and }}$ exhibited an incidence of late AMD of $12 \%$.

The main limitations of this study were the low number of events making it impossible to investigate AMD subtypes. In addition, our model tended to underestimate the incidence of advanced AMD for the high-risk groups, particularly in the validation set, which is most probably due to the higher incidence rates in ALIENOR compared to RS-I. As discussed previously, these differences in incidence rates are partially due to age differences among the cohorts ${ }^{42}$, but may also be due to differences in design, classification of disease and potentially differences in AMD incidence among the Netherlands and France. Further validation in other cohorts, in particular in populations with a different genetic make-up (such as Asian populations), would enhance the generalizability of this model.

Further enhancements of this prediction model could still be achieved by integrating multiple data sources as color fundus photographs (CFPs) and Optical Coherence Tomography (OCT) examinations. Indeed, in the present model, description of phenotype is based on human interpretation of a few standard retinal alterations on

a supprimé: , while the performance at long term appeared somewhat lower ( $88 \%$ versus $91 \%$ at 15 years, in the Rotterdam study). 
CFPs (large drusen and pigmentary abnormalities composing the AREDS simplified score, together with intermediate drusen and hyperpigmentation). Automated grading of retinal images using deep learning methods can improve the prediction accuracy of the model compared to manual grading which is subjective and time-consuming. ${ }^{45-}$ 49 Moreover, previous studies have suggested that SD-OCT parameters, such as drusen volume, drusen reflectivity, presence of hyperreflective foci or retinal thickness, may improve prediction of progression to advanced AMD. ${ }^{46,50-52}$ In conclusion, this prediction model for advanced AMD based on a wide set of risk factors achieved high discrimination performances. It will be made available to ophthalmologists and patients through the website www.macutest.net. The approval by the U.S. Food and Drug Administration of devices based on artificial intelligence highlights the potential of combining such prediction models - based on $\mathrm{ML}$ techniques - with ophthalmologists' expertise to suggest personalized recommendations and to lower the risk of AMD. Our prediction model paves the way towards making precision medicine for AMD patients a reality in the near future.

\section{References}

1. Bourne RR, Stevens GA, White RA, et al. Causes of vision loss worldwide, 1990-2010: a systematic analysis. Lancet Glob Health 2013;1:e339-349.

2. Mitchell P, Liew G, Gopinath B, Wong TY. Age-related macular degeneration. Lancet 2018;392:1147-1159.

3. Colijn JM, Buitendijk GHS, Prokofyeva E, et al. Prevalence of Age-Related Macular Degeneration in Europe: The Past and the Future. Ophthalmology 2017;124:1753-1763.

4. Wong WL, Su X, Li X, et al. Global prevalence of age-related macular degeneration and disease burden projection for 2020 and 2040: a systematic review and meta-analysis. Lancet Glob Health 2014;2:e106-116.

5. Erie JC, Barkmeier AJ, Hodge DO, Mahr MA. High Variation of Intravitreal Injection Rates and Medicare Anti-Vascular Endothelial Growth Factor Payments per Injection in the United States. Ophthalmology 2016;123:1257-1262. 
6. Pylypchuk R, Wells S, Kerr A, et al. Cardiovascular disease risk prediction equations in 400000

419 primary care patients in New Zealand: a derivation and validation study. Lancet 2018;391:1897-1907.

420 7. Sobrin L, Seddon JM. Nature and nurture- genes and environment- predict onset and

421 progression of macular degeneration. Prog Retin Eye Res 2014;40C:1-15.

8. Seddon JM, Reynolds R, Maller J, et al. Prediction model for prevalence and incidence of advanced age-related macular degeneration based on genetic, demographic, and environmental variables. Invest Ophthalmol Vis Sci 2009;50:2044-2053.

9. Seddon JM, Reynolds R, Yu Y, et al. Risk models for progression to advanced age-related macular degeneration using demographic, environmental, genetic, and ocular factors. Ophthalmology 2011;118:2203-2211.

10. Buitendijk GH, Rochtchina E, Myers C, et al. Prediction of age-related macular degeneration in the general population: the Three Continent AMD Consortium. Ophthalmology 2013;120:26442655.

11. Chiu CJ, Mitchell P, Klein R, et al. A risk score for the prediction of advanced age-related macular degeneration: development and validation in 2 prospective cohorts. Ophthalmology 2014;121:1421-1427.

12. Seddon JM, Rosner B. Validated Prediction Models for Macular Degeneration Progression and Predictors of Visual Acuity Loss Identify High-Risk Individuals. Am J Ophthalmol 2019;198:223261.

13. Chen Y, Zeng J, Zhao C, et al. Assessing susceptibility to age-related macular degeneration with genetic markers and environmental factors. Arch Ophthalmol 2011;129:344-351.

14. Klein ML, Francis PJ, Ferris FL, 3rd, et al. Risk assessment model for development of advanced age-related macular degeneration. Arch Ophthalmol 2011;129:1543-1550.

15. Breiman L. Heuristics of Instability and Stabilization in Model Selection. The Annals of Statistics 1996;24:2350-2383.

16. Heinze G, Wallisch C, Dunkler D. Variable selection - A review and recommendations for the practicing statistician. Biometrical Journal 2018;60:431-449.

17. Mansiaux $Y$, Carrat $F$. Detection of independent associations in a large epidemiologic dataset: a comparison of random forests, boosted regression trees, conventional and penalized logistic regression for identifying independent factors associated with H1N1pdm influenza infections. BMC Med Res Methodol 2014;14:99.

18. Obermeyer Z, Emanuel EJ. Predicting the Future - Big Data, Machine Learning, and Clinical Medicine. N Engl J Med 2016;375:1216-1219.

19. Leffondre $\mathrm{K}$, Touraine $\mathrm{C}$, Helmer $\mathrm{C}$, Joly $\mathrm{P}$. Interval-censored time-to-event and competing risk with death: is the illness-death model more accurate than the Cox model? Int J Epidemiol 2013;42:1177-1186.

20. Ikram MA, Brusselle GGO, Murad SD, et al. The Rotterdam Study: 2018 update on objectives, design and main results. Eur J Epidemiol 2017;32:807-850.

21. Delcourt C, Korobelnik JF, Barberger-Gateau P, et al. Nutrition and Age-Related Eye Diseases: The ALIENOR (Antioxydants, Lipides Essentiels, Nutrition et Maladies Oculaires) Study. J Nutr Health Aging 2010;14:854-861.

22. Klein R, Davis MD, Magli YL, et al. The Wisconsin age-related maculopathy grading system. Ophthalmology 1991;98:1128-1134.

23. Bird AC, Bressler NM, Bressler SB, et al. An international classification and grading system for age-related maculopathy and age-related macular degeneration. The International ARM Epidemiological Study Group. Surv Ophthalmol 1995;39:367-374.

24. Holm S. A Simple Sequentially Rejective Multiple Test Procedure. Scandinavian Journal of Statistics 1979;6:65-70.

25. Ferris FL, Davis MD, Clemons TE, et al. A simplified severity scale for age-related macular degeneration: AREDS Report No. 18. Arch Ophthalmol 2005;123:1570-1574.

26. Ferris FL, 3rd, Wilkinson CP, Bird A, et al. Clinical classification of age-related macular degeneration. Ophthalmology 2013;120:844-851. 
27. Van Leeuwen R, Klaver CC, Vingerling JR, et al. The risk and natural course of age-related maculopathy: follow-up at $61 / 2$ years in the Rotterdam study. Arch Ophthalmol 2003;121:519-526. 28. Sofi F, Macchi C, Abbate R, et al. Mediterranean diet and health status: an updated metaanalysis and a proposal for a literature-based adherence score. Public Health Nutr 2014;17:27692782.

29. Fritsche LG, Ig| W, Bailey JN, et al. A large genome-wide association study of age-related macular degeneration highlights contributions of rare and common variants. Nat Genet 2016;48:134143.

30. Tibshirani R. The lasso method for variable selection in the Cox model. Stat Med 1997;16:385-395.

31. Zou H, Hastie T. Regularization and variable selection via the elastic net. Journal of the Royal Statistical Society: Series B (Statistical Methodology) 2005;67:301-320.

32. Zou H. The Adaptive Lasso and Its Oracle Properties. Journal of the American Statistical Association 2006;101:1418-1429.

33. Bach FR. Bolasso: model consistent Lasso estimation through the bootstrap. Proceedings of the 25th international conference on Machine learning. Helsinki, Finland: ACM, 2008.

34. Breiman L. Bagging Predictors. Machine Learning 1996;24:123-140.

35. Cattell RB. The Scree Test For The Number Of Factors. Multivariate Behav Res 1966;1:245-

276.

36. Touraine C, Helmer C, Joly P. Predictions in an illness-death model. Stat Methods Med Res 2016;25:1452-1470.

37. Touraine C, Gerds TA, Joly P. SmoothHazard: An R Package for Fitting Regression Models to Interval-Censored Observations of Illness-Death Models. Journal of Statistical Software 2017;79:1-22. 38. Delcourt C, Souied E, Sanchez A, Bandello F. Development and Validation of a Risk Score for Age-Related Macular Degeneration: The STARS Questionnaire. Invest Ophthalmol Vis Sci 2017;58:6399-6407.

39. Blanche P, Dartigues JF, Jacqmin-Gadda H. Estimating and comparing time-dependent areas under receiver operating characteristic curves for censored event times with competing risks. Stat Med 2013;32:5381-5397.

40. van Buuren S, Groothuis-Oudshoorn K. mice: Multivariate Imputation by Chained Equations in R. Journal of Statistical Software; Vol 1, Issue 3 (2011) 2011.

41. Seddon JM, Silver RE, Kwong M, Rosner B. Risk Prediction for Progression of Macular Degeneration: 10 Common and Rare Genetic Variants, Demographic, Environmental, and Macular Covariates. Invest Ophthalmol Vis Sci 2015;56:2192-2202.

42. Merle BMJ, Colijn JM, Cougnard-Gregoire A, et al. Mediterranean Diet and Incidence of Advanced Age-Related Macular Degeneration: The EYE-RISK Consortium. Ophthalmology 2019;126:381-390.

43. Cougnard-Gregoire A, Delyfer MN, Korobelnik JF, et al. Long-Term Blood Pressure and AgeRelated Macular Degeneration: The ALIENOR Study. Invest Ophthalmol Vis Sci 2013;54:1905-1912. 44. Areds. Risk factors associated with age-related macular degeneration. A case-control study in the age-related eye disease study: age-related eye disease study report number 3. Age-Related Eye Disease Study Research Group. Ophthalmology 2000;107:2224-2232.

45. Peng Y, Dharssi S, Chen Q, et al. DeepSeeNet: A Deep Learning Model for Automated Classification of Patient-based Age-related Macular Degeneration Severity from Color Fundus Photographs. Ophthalmology 2019;126:565-575.

46. Schmidt-Erfurth U, Waldstein SM, Klimscha S, et al. Prediction of Individual Disease Conversion in Early AMD Using Artificial Intelligence. Invest Ophthalmol Vis Sci 2018;59:3199-3208.

47. De Fauw J, Ledsam JR, Romera-Paredes B, et al. Clinically applicable deep learning for diagnosis and referral in retinal disease. Nature medicine 2018;24:1342-1350.

48. Burlina PM, Joshi N, Pekala M, et al. Automated Grading of Age-Related Macular Degeneration From Color Fundus Images Using Deep Convolutional Neural Networks. JAMA Ophthalmol 2017;135:1170-1176. 
49. Burlina PM, Joshi N, Pacheco KD, et al. Use of Deep Learning for Detailed Severity Characterization and Estimation of 5-Year Risk Among Patients With Age-Related Macular Degeneration. JAMA Ophthalmol 2018;136:1359-1366.

50. Hallak JA, de Sisternes L, Osborne A, et al. Imaging, Genetic, and Demographic Factors Associated With Conversion to Neovascular Age-Related Macular Degeneration: Secondary Analysis of a Randomized Clinical Trial. JAMA Ophthalmol 2019;137:738-744.

51. Ferrara D, Silver RE, Louzada RN, et al. Optical Coherence Tomography Features Preceding the Onset of Advanced Age-Related Macular Degeneration. Invest Ophthalmol Vis Sci 2017;58:35193529.

52. Waldstein SM, VogI WD, Bogunovic $\mathrm{H}$, et al. Characterization of Drusen and Hyperreflective Foci as Biomarkers for Disease Progression in Age-Related Macular Degeneration Using Artificial Intelligence in Optical Coherence Tomography. JAMA Ophthalmol 2020.

\section{Figures legends:}

Figure 1. The bootstrap lasso and IDM procedures for developing the prediction model of advanced AMD.

Figure 2. Flowchart of Rotterdam Study I (RS-I) and Antioxydants, LIpids Essentiels, Nutrition et maladies OculaiRes (ALIENOR) study.

Figure 3. Selection frequency of variables in the Rotterdam Study I (RS-I) using the Bolasso method.

The vertical dashed line represent the threshold corresponding to a selection frequency equals to $80 \%$. 
Figure 4. Cumulative incidence of advanced age-related macular degeneration (AMD) according to risk categories.

A, Rotterdam Study I (RS-I). B, Antioxydants, LIpids Essentiels, Nutrition et maladies OculaiRes (ALIENOR) study.

Figure 5. Calibration of prediction risk scores per risk group.

A,B,C. Comparison of the cross-validated predicted and observed cumulative incidence in the Rotterdam Study I (RS-I). D. Comparison of the predicted and observed cumulative incidence in the Antioxydants, LIpids Essentiels, Nutrition et maladies OculaiRes (ALIENOR) study.

The vertical bars represent the $95 \%$ confidence intervals of the observed cumulative incidences.

\section{Annex:}

Members of the Eye-Risk consortium: Erkin I. Acar, MSc (Department of Ophthalmology, Radboud University Medical Center, Nijmegen, Netherlands), Blanca Arango-Gonzalez, M.D. (Centre for Ophthalmology, Institute for Ophthalmic Research, Eberhard Karls University Tübingen, University Clinic Tübingen, Tübingen, Germany); Angela Armento, $\mathrm{PhD}$ (Centre for Ophthalmology, Institute for Ophthalmic Research, Eberhard Karls University Tübingen, University Clinic Tübingen, Tübingen, Germany); Franz Badura (PRO RETINA Deutschland e.V.); Vaibhav Bhatia, PhD (Department of Regeneration and Cell Therapy, Andalusian Molecular Biology and 
Regenerative Medicine Centre (CABIMER), Seville, Spain); Shomi S. Bhattacharya, PhD (Department of Regeneration and Cell Therapy, Andalusian Molecular Biology and Regenerative Medicine Centre (CABIMER), Seville, Spain); Marc Biarnés, PhD (Barcelona Macula Foundation, Barcelona, Spain); Anna Borrell, MSc (Barcelona Macula Foundation, Barcelona, Spain); Sofia M. Calado, PhD (Department of Regeneration and Cell Therapy, Andalusian Molecular Biology and Regenerative Medicine Centre (CABIMER), Seville, Spain); Sascha Dammeier, PhD (Centre for Ophthalmology, Institute for Ophthalmic Research, Eberhard Karls University Tübingen, University Clinic Tübingen, Tübingen, Germany); Anita de Breuk, M.D. (Department of Ophthalmology, Radboud University Medical Center, Nijmegen, Netherlands); Berta De la Cerda, PhD (Department of Regeneration and Cell Therapy, Andalusian Molecular Biology and Regenerative Medicine Centre (CABIMER), Seville, Spain); Anneke I. den Hollander, PhD (Department of Ophthalmology, Radboud University Medical Center, Nijmegen, Netherlands, Department of Human Genetics, Radboud University Medical Center, Nijmegen, Netherlands); Francisco J. Diaz- Corrales, M.D., PhD (Department of Regeneration and Cell Therapy, Andalusian Molecular Biology and Regenerative Medicine Centre (CABIMER), Seville, Spain); Sigrid Diether, PhD (Centre for Ophthalmology, Institute for Ophthalmic Research, Eberhard Karls University Tübingen, University Clinic Tübingen, Tübingen, Germany); Eszter Emri, PhD (Centre for Experimental Medicine, Queen's University Belfast, Belfast, United Kingdom); Tanja Endermann, PhD (Assay Development, AYOXXA Biosystems GmbH, Cologne, Germany); Lucia L. Ferraro, M.D. (Barcelona Macula Foundation, Barcelona, Spain); Míriam Garcia, OD, MSc (Barcelona Macula Foundation, Barcelona, Spain); Thomas J. Heesterbeek, M.D. (Department of Ophthalmology, Radboud University Medical 
Center, Nijmegen, Netherlands); Sabina Honisch, PhD (Centre for Ophthalmology, Institute for Ophthalmic Research, Eberhard Karls University Tübingen, University Clinic Tübingen, Tübingen, Germany); Carel B. Hoyng, M.D. (Department of Ophthalmology, Radboud University Medical Center, Nijmegen, Netherlands); Ellen Kilger, PhD (Centre for Ophthalmology, Institute for Ophthalmic Research, Eberhard Karls University Tübingen, University Clinic Tübingen, Tübingen, Germany); Elod Kortvely, PhD (Roche Innovation Center Basel); Claire Lastrucci, PhD (Centre for Genomic Regulation, Barcelona, Spain); Hanno Langen, PhD (Roche Innovation Center Basel, F. Hoffmann-La Roche Ltd, Basel, Switzerland); Imre Lengyel, PhD (Centre for Experimental Medicine, Queen's University Belfast, Belfast, United Kingdom); Phil Luthert, PhD (Institute of Ophthalmology, University College London, London, United Kingdom); Jordi Monés, MD, PhD (Barcelona Macula Foundation, Barcelona, Spain); Everson Nogoceke, PhD (Roche Innovation Center Basel, F. Hoffmann-La Roche Ltd, Basel, Switzerland); Tunde Peto, M.D., PhD (Centre for Public Health, Queen's University Belfast, Belfast, United Kingdom); Frances M. Pool, PhD (Ocular Biology, UCL Institute of Opthalmology, London, United Kingdom); Eduardo Rodriguez-Bocanegra, MSc (Barcelona Macula Foundation, Barcelona, Spain); Luis Serrano, PhD (Centre for Genomic Regulation, Barcelona, Spain); Jose Sousa, PhD (Advanced Informatics CTU, Queen's University Belfast, Belfast, United Kingdom); Eric Thee, M.D. (Department of Epidemiology, Erasmus Medical Center, Rotterdam, Netherlands, Department of Ophthalmology, Erasmus Medical Center, Rotterdam, Netherlands); Marius Ueffing, $\mathrm{PhD}$ (Centre for Ophthalmology, Institute for Ophthalmic Research, Eberhard Karls University Tübingen, University Clinic Tübingen, Tübingen, Germany, Department of Ophthalmology, University MedicalCentre Tübingen, Tübingen, Germany); Karl U. Ulrich Bartz-Schmidt, M.D. 
